\title{
Anais do Congresso Acervo+ 2022 | Eixo Multi
}

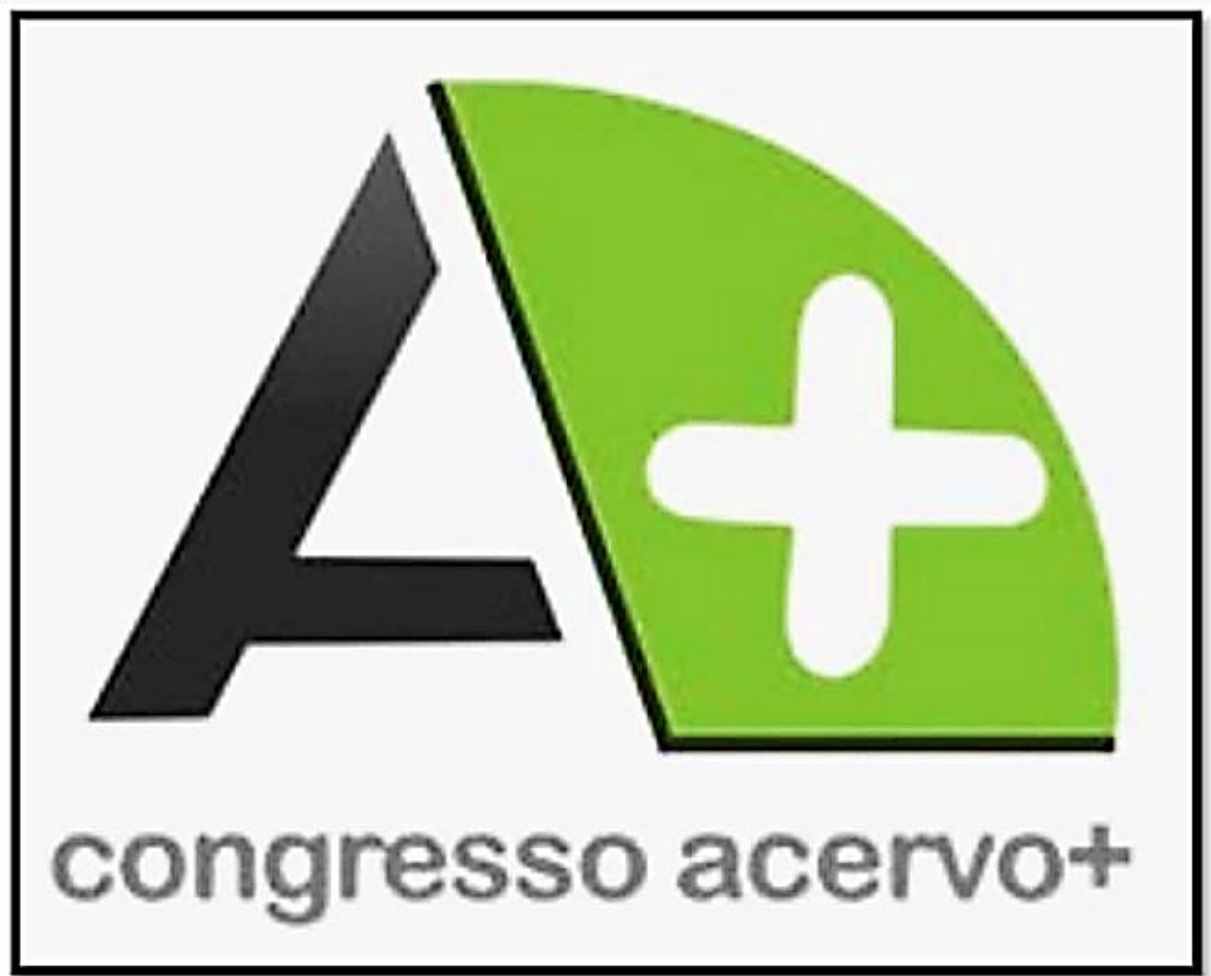

Apoio:

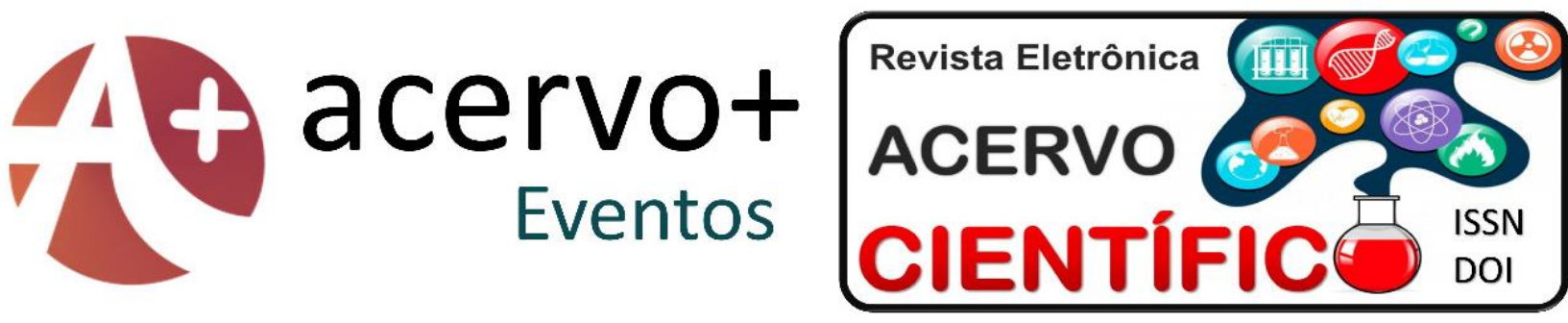




\section{SUMÁRIO}

SOBRE O EVENTO 3

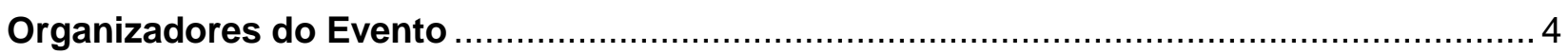

Comissão Científica

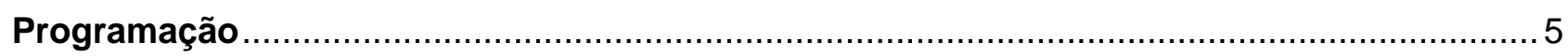

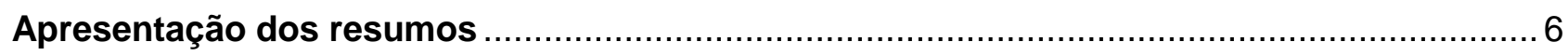

RESUMOS SIMPLES

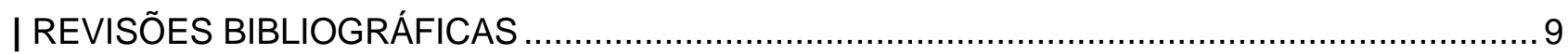

ESPAÇOS PÚBLICOS: UMA REVISÃO INTEGRATIVA SOBRE AS ESTRATÉGIAS URBANÍSTICAS EM TEMPOS DE COVID-19 ...............................................................

O IMPACTO DA PANDEMIA NA SAÚdE EMOCIONAL DAS CRIANÇAS: REVISÃo

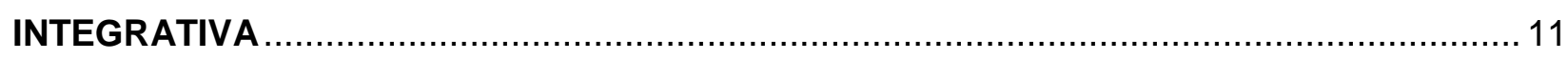

OS DESAFIOS DA IMPLEMENTAÇÃO DO SANEAMENTO BÁSICO NO BRASIL .................13

O DESENVOLVIMENTO NEUROPSICOMOTOR E A INFLUÊNCIA DE RISCOS BIOLÓGICOS

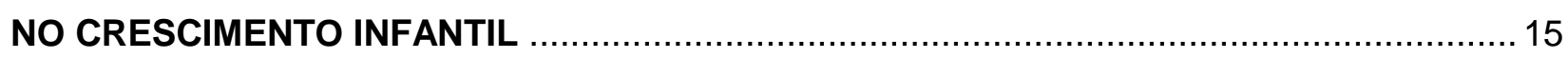

CONHECIMENTO DOS UNIVERSITÁRIOS ACERCA DA PANDEMIA DA COVID-19: UMA REVISÃO INTEGRATIVA DA LITERATURA

POTENCIAL TECNOLÓGICO DAS NANOPARTÍCULAS MAGNÉTICAS COMO PRÁTICA

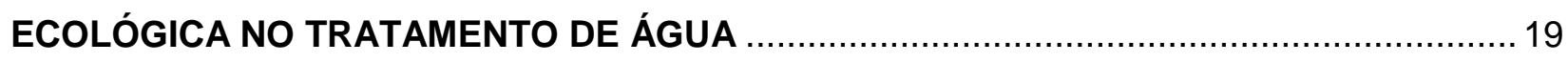

ENTENDENDO O FENÔMENO RELIGIOSO SEGUNDO VIKTOR FRANKL ...........................21

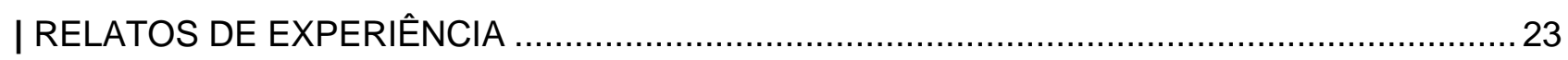

IMPLANTAÇÃO DO CURSO DE PEDAGOGIA MOdALIDADE A DISTÂNCIA PELA UNIVERSIDADE FEDERAL DO TRIÂNGULO MINEIRO: UM RELATO DE EXPERIÊNCIA ... 23 A APLICABILIDADE RENTÁVEL DAS ESTACAS PRÉ-MOLdADAS DE CONCRETO

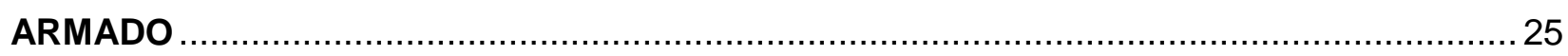

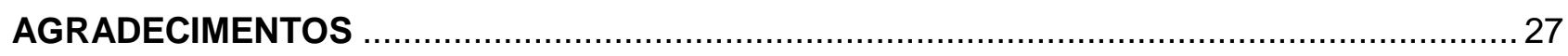




\section{SOBRE O EVENTO}

O Congresso Acervo+ mantém o seu propósito de contribuir para a disseminação da ciência. Através dos cadernos de anais publicados na Revista Eletrônica Acervo Saúde (REAS) e na Revista Eletrônica Acervo Científico (REAC), a sociedade é agraciada com um rico material científico de diversas áreas do conhecimento.

A organização enfrentou novos desafios para realizar mais uma edição de sucesso do evento, que contasse com uma programação robusta e proveitosa aos participantes, além da publicação de um caderno de anais de qualidade. A partir da inovação, um dos pilares da Acervo+, os desafios foram superados.

Antes mesmo do anúncio oficial da data do evento, os autores contaram com a assistência de uma masterclass com sete aulas, esclarecendo todos os pontos a respeito da construção de um resumo científico. Além disso, o processo de submissão foi reestruturado e se tornou intuitivo e didático aos autores, visando garantir que erros comuns fossem evitados nos resumos.

As apresentações orais, por meio do evento ao vivo no YouTube, e dos episódios de podcast, garantem acessibilidade e inovação na disseminação da ciência. Em mais um evento, a Acervo+ possibilita que pessoas de todo o Brasil e do mundo fortaleçam a pesquisa e iniciem sua trajetória científica.

Para garantir uma programação de excelência, a organização estruturou palestra, mesa-redonda, relato de experiência e aula-aberta divididos entre os quatro dias de Congresso. Além disso, a Oficina do Artigo foi uma novidade da edição de 2022, um convite para que os autores avancem na pesquisa e atinjam novos objetivos e metas, como a publicação de um artigo em periódico.

Acervo+ conectando você ao universo científico!

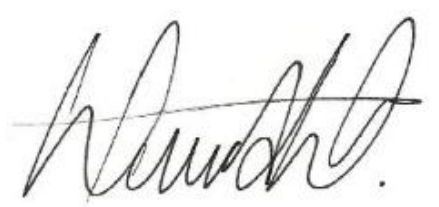

Dr. Andreazzi Duarte editor-líder 


\section{Organizadores do Evento}

- Equipe Editorial de Anais de Eventos Acervo+

- Equipe de Comunicação Acervo+

- Equipe Administrativa Acervo+

\section{Comissão Científica}

- Equipe Editorial de Anais de Eventos Acervo+

- Equipe Editorial de Artigos Acervo+ 


\section{Programação}

\begin{tabular}{|c|c|c|}
\hline \multicolumn{3}{|c|}{ 1ㅇ DIA - 24/01/2022 } \\
\hline Horário & Evento & Local \\
\hline $19 h 00$ & Cerimônia de abertura & \multirow{4}{*}{ YouTube } \\
\hline $19 h 10$ & Aula: Publicação científica na graduação, é possível? & \\
\hline $20 \mathrm{~h} 00$ & Premiação Impactos A+ de 2021 & \\
\hline $21 \mathrm{~h} 00$ & Encerramento do $1^{\circ}$ dia & \\
\hline \multicolumn{3}{|c|}{$2^{\circ}$ DIA - 25/01/2022 } \\
\hline Horário & Evento & Local \\
\hline $19 \mathrm{~h} 00$ & Início do $2^{\circ}$ dia & \multirow{6}{*}{ YouTube } \\
\hline $19 h 05$ & Palestra: Gestão e liderança hospitalar & \\
\hline $20 \mathrm{~h} 00$ & Introdução à Oficina do Artigo (1ª parte) & \\
\hline $20 h 40$ & Intervalo & \\
\hline $20 h 45$ & Apresentação de 10 trabalhos & \\
\hline $22 \mathrm{~h} 00$ & Encerramento do $2^{\circ}$ dia & \\
\hline \multicolumn{3}{|c|}{ 3 DIA - 26/01/2022 } \\
\hline Horário & Evento & Local \\
\hline $19 \mathrm{~h} 00$ & Início do 3ํ dia & \multirow{6}{*}{ YouTube } \\
\hline 19h05 & Mesa-redonda: Saúde mental e a Logoterapia na pós-pandemia & \\
\hline $20 \mathrm{~h} 00$ & Introdução à Oficina do Artigo ( (2ª parte) & \\
\hline $20 \mathrm{~h} 40$ & Intervalo & \\
\hline $20 h 45$ & Apresentação de 10 trabalhos & \\
\hline $22 \mathrm{~h} 00$ & Encerramento do $3^{\circ}$ dia & \\
\hline \multicolumn{3}{|c|}{$4^{\circ}$ DIA - 27/01/2022 } \\
\hline Horário & Evento & Local \\
\hline $19 \mathrm{~h} 00$ & Início do 4º dia & \multirow{6}{*}{ YouTube } \\
\hline $19 \mathrm{~h} 10$ & Relato de experiência: Empreendedorismo em saúde & \\
\hline $20 \mathrm{~h} 00$ & Intervalo & \\
\hline $20 \mathrm{~h} 05$ & Apresentação de 10 trabalhos & \\
\hline $21 \mathrm{~h} 30$ & Premiação & \\
\hline $21 \mathrm{~h} 40$ & Cerimônia de encerramento & \\
\hline
\end{tabular}




\section{Apresentação dos resumos}

Mais de 400 resumos foram enviados por autores de todo o Brasil e exterior para o Congresso Acervo+. A submissão, nesta edição do evento, foi feita por meio de uma nova plataforma, didática e intuitiva, que orientava os autores a respeito de elementos que não estivessem de acordo com a diretriz da revista.

Cada processo foi avaliado individualmente pela equipe editorial da Revista Eletrônica Acervo Saúde (REAS) e da Revista Eletrônica Acervo Científico (REAC), que emitia o parecer favorável ou não à publicação. Todos os resumos que não atendiam às normas dos periódicos receberam oportunidade de correção e reenvio.

Dentre os critérios utilizados para a avaliação dos resumos estão:

1. Concisão e fidedignidade textual;

2. Impacto, atualidade e originalidade;

3. Dados preliminares por fontes confiáveis;

4. Acessibilidade e clareza;

5. Delineamento adequado da pesquisa;

6. Ética em pesquisa;

7. Definição clara dos objetivos, resultados e variáveis do estudo;

8. Narrativa com fluidez e linguagem adequada;

9. Didática e coerência de raciocínio e percurso;

10. Aplicação, informação e/ou conhecimento no âmbito científico.

Após a avaliação, 290 resumos foram considerados aptos à publicação. Destes, 281 dispõem de temáticas da área da saúde, sendo direcionados para publicação na REAS. $O$ caderno é composto de 52 estudos originais, 195 revisões bibliográficas, quatro estudos de caso e 30 relatos de experiência. Outros nove resumos do eixo multidisciplinar foram direcionados para publicação na REAC, sendo sete revisões bibliográficas e dois relatos de experiência.

A avaliação feita pela equipe editorial também solicitou e verificou a documentação pertinente de cada resumo, incluindo os Termos de Consentimento Livre e Esclarecido (TCLEs) dos estudos de caso e os pareceres do Comitê de Ética em Pesquisa (CEP) para 
os estudos originais. Quando necessário, outros documentos também foram solicitados durante a auditoria de cada processo. 


\section{RESUMOS SIMPLES}

Revisão Bibliográfica: 07 resumos

Relato de Experiência: 02 resumos 


\title{
| REVISÕES BIBLIOGRÁFICAS
}

RESUMO SIMPLES: Revisão Integrativa

\section{ESPAÇOS PÚBLICOS: UMA REVISÃO INTEGRATIVA SOBRE AS ESTRATÉGIAS URBANÍSTICAS EM TEMPOS DE COVID-19}

\author{
Marina Lima Figueirêdo ${ }^{1}$ \\ Kalênio Sergio Vilar F. Dantas ${ }^{1}$ \\ Cristiane Augusta Gomes Bodra ${ }^{1}$ \\ Josino Gomes da Silva Neto ${ }^{1}$ \\ Alberlene Baracho Sales ${ }^{2}$
}

1. Centro Universitário de João Pessoa (UNIPE), João Pessoa - PB.

2. Universidade Federal da Paraíba (UFPB), João Pessoa - PB.

Palavras-chave: Espaços públicos, Covid-19, Urbanismo.

\section{INTRODUÇÃO}

O ano de 2020 foi marcado pelo contexto pandêmico de Covid-19, que condicionou a população mundial a um distanciamento social (NECA BR e RECHIA S, 2020). Esse distanciamento foi necessário como medida de prevenção, objetivando a diminuição de casos de contaminação pelo vírus da Covid-19. Em meio a toda essa situação de isolamento social e sentimento de apreensão, que possibilitou ver a vida sob uma nova ótica. Nos encontramos vulneráveis a um vírus, outrora desconhecido, que restringiu as pessoas ao convívio, social e com as paisagens naturais que traziam bem-estar, qualidade de vida e a sensação de pertencimento.

\section{OBJETIVO}

Revisar as estratégias referentes a requalificação dos espaços públicos urbanos, considerando os pontos estratégicos de vivência da cidade mediante o contexto pandêmico que se vivenciado nos anos de 2020 e 2021.

\section{MÉTODO}

A metodologia de revisão integrativa se propõe a realizar uma análise dos resultados de modo a sintetizar a amostragem. Essa síntese buscou artigos nas plataformas Scielo e Google acadêmico, utilizando os descritores: urbanismo, covid-19, estratégias, espaços públicos, sendo selecionados 2 artigos dos 11 encontrados sobre o tema. Buscou-se ainda como critério de inclusão apenas pesquisas de campo, excluindo revisões de literatura. 


\section{REVISÃO BIBLIOGRÁFICA}

A assimetria das realidades urbanas foi mais acentuada nos espaços públicos durante a pandemia. As estratégias de recuperação, redesenho ou programação dos espaços, concede um retorno gradual ao uso desses espaços. A implementação de ciclofaixas caracteriza-se como uma ferramenta importante para a vivência da cidade em tempos de covid-19 (ROCHA GQ, 2021).

O baixo investimento estatal no planejamento urbano promove a desigualdade e vulnerabilidade social nos espaços públicos. Dentre as medidas de retorno aos espaços públicos foram inseridos equipamentos urbanos que disponibilizam acesso a higienização das mãos, como pias comunitárias, e materiais de divulgação em redes sociais para promover a conscientização sobre o covid-19 (dos SANTOS GJ e dos SANTOS LMM, 2021).

\section{CONSIDERAÇÕES FINAIS}

Este trabalho aponta que a pandemia influenciou diretamente a requalificação dos espaços públicos urbanos, intensificando a assimetria das vivências urbanas e transparecendo a falta de investimento estatal na infraestrutura urbana. No entanto, para o retorno a esses locais, foram exigidas medidas preventivas a covid-19, como a implementação de equipamentos que possibilitam a higienização das mãos e melhorias nas ciclofaixas.

\section{REFERÊNCIAS}

1. NECA BR, RECHIA S. Ficar em Casa ou Ocupar os Espaços de Lazer ao Ar Livre?: Reflexões e Possibilidades para uma Apropriação Segura dos Diferentes Espaços Públicos de Lazer em Tempos de Pandemia. LICERE-Revista do Programa de Pós-graduação Interdisciplinar em Estudos do Lazer, 2020; 23(4): 471-509.

2. ROCHA GQ. Coautoria urbana e quarentena: relações pessoa-cidade na pandemia do novo coronavírus. Cadernos Metrópole, 2021; 23: 1017-1038.

3. dos SANTOS GJ, dos SANTOS LMM. Reflexões sobre a relação pessoa-ambiente e os espaços públicos em tempos de Pandemia de COVID-19. Saúde Mental no Século XXI Indivíduo e Coletivo Pandêmico, $2021 ; 244-258$. 
RESUMO SIMPLES: Revisão Integrativa

\title{
O IMPACTO DA PANDEMIA NA SAÚDE EMOCIONAL DAS CRIANÇAS: REVISÃO INTEGRATIVA
}

\author{
Franciely Alves da Silva ${ }^{1}$ \\ Jéssica Priscila Fragoso de Moura² \\ Wilza Candida Santos e Silva ${ }^{3}$ \\ Marlene Alves Palmeira dos Santos ${ }^{4}$ \\ Lorena Silva Freire 5
}

1. Universidade Federal do Triângulo Mineiro (UFTM), Uberaba - MG.

2. Universidade Estadual de Campinas (UNICAMP), Limeira - SP.

3. Instituto Federal de Educação, Ciência e Tecnologia de São Paulo (IFSP), São Paulo - SP.

4. Centro Universitário do Vale do Araguaia, Barra do Garças - MT.

5. Universidade de Cuiabá (UNIC), Rondonópolis - MT.

Palavras-chave: Crianças, Pandemia, Saúde mental.

\section{INTRODUÇÃO}

Coronavirus Disease (COVID-19) se originou pelo Severe Acute Respiratory Syndrome Coronavirus 2 (SARS-CoV-2) na China e logo se tornou uma pandemia alastrando por 188 países. Apesar da incidência da COVID-19 ser menor entre as crianças, a pandemia ocasionou transtornos na saúde mental da mesma, devido a recomendação do distanciamento social. Assim como os adultos, as crianças tiveram suas rotinas alteradas devido a essa pandemia ausentando-se da interação da escola, brincadeiras, passeios e afins. Essas mudanças podem negativamente impactar na saúde mental das crianças, repercutindo em outras fases do desenvolvimento humano (AYDOGDU ALF, 2020; LADEIA DN, et al., 2020).

\section{OBJETIVO}

Revisar e investigar, por meio de uma revisão de literatura integrativa, o impacto da pandemia ocasionado pelo novo Coronavírus causador da doença COVID-19 na saúde mental das crianças, salientando os fatores que causam esses danos.

\section{REVISÃO BIBLIOGRÁFICA}

A pandemia afeta negativamente a saúde mental infantil e os principais comportamentos encontrados são: medo, ansiedade, estresse, desânimo, tristeza, preocupação, raiva, inquietude, insônia, sentimentos de desamparo e sofrimento, além de apresentar comportamentos agressivos e desrespeitosos (AYDOGDU ALF, 2020). 
O principal fator que afeta negativamente a saúde mental das crianças é a alteração comportamental dos pais como irritabilidade em decorrência a mudanças em sua rotina. Crianças que vivenciam alteração de comportamentos dos pais apresentam uma redução na qualidade de sono, sensação de desamparo e estresse (JOHNSTON J, et al., 2020). Dessa forma, é necessário pensar criticamente no pós-pandemia, para que as medidas sejam realizadas para tratar os traumas e as sequelas futuras que podem gerar nas crianças.

\section{CONSIDERAÇÕES FINAIS}

A pandemia afeta a saúde mental das crianças, entre os principais comportamentos são: e tristeza, medo, ansiedade, insônia, raiva e estresse. Dessa forma, é necessário que medidas de preservação da saúde mental das crianças agora sejam realizadas para não ocasionar danos mentais futuro quando adultos.

\section{REFERÊNCIAS}

1. AYDOGDU ALF. Saúde mental das crianças durante a pandemia causada pelo novo coronavírus: revisão integrativa. Journal Health Npeps, 2020; 2: 1-17.

2. JOHNSTON J, et al. Perfectionism is associated with higher eating disorder symptoms and lower remission in children and adolescents diagnosed with eating disorders. Eating behaviors, 2018; 30: 55-60.

3. LADEIA DN, et al. Análise da saúde mental na população geral durante a pandemia de Covid-19. Revista Eletrônica Acervo Saúde, 2020; Esp.46: e3925. 


\section{OS DESAFIOS DA IMPLEMENTAÇÃO DO SANEAMENTO BÁSICO NO BRASIL}

Anderson Alves de Aguiar ${ }^{1}$

1. Faculdade Maurício de Nassau (UNINASSAU), Parnaíba - PI.

Palavras-chave: Saneamento básico, Legislação, Desafios.

\section{INTRODUÇÃO}

O saneamento básico pode ser conceituado como o conjunto de ações efetivadas entre sociedade e meio ambiental com a finalidade de beneficiar ambos e oportunizar questões como saúde, qualidade de vida e desenvolvimento (MELLO MTC e MELLO LTC, 2019). No Brasil, segundo dados do Sistema Nacional de Informações sobre Saneamento (SNIS) aproximadamente metade da população não usufrui de esgotamento sanitário, e somente $46 \%$ dos sistemas de esgotos existentes são tratados. E entre outros números, um pouco mais de $16 \%$ da população não obtêm água tratada, o que demonstra que o cenário brasileiro apresenta ainda muitas dificuldades (BRASIL, 2019).

\section{OBJETIVO}

Revisar trabalhos de natureza científica sobre problemas, dificuldades e implantação do saneamento básico no Brasil, buscando compreender o estado atual e configurações de saneamento e implementação de políticas públicas nesse contexto, como a Lei Nacional do Saneamento Básico.

\section{REVISÃO BIBLIOGRÁFICA}

Ao longo dos anos o Brasil passou por diversas transformações e desde seus primeiros períodos foram dados os primeiros passos em busca do correto manejo de esgotos e águas pluviais (COSTA RNP e PINHEIRO EM, 2018). Atualmente entre os maiores desafios para a efetivação do saneamento estão a conscientização social e a falta de investimentos para promoção de saneamento básico, que leva infraestrutura para a população (MELLO MTC e MELLO LTC, 2019).

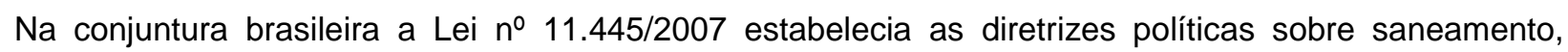
propondo ditar distribuição de água potável, limpeza e manejo de resíduos e drenagem, e esgotamento sanitário. Não obstante foi proposta, mais recentemente, a Lei ํㅡ 14.026/2020, atualizando a legislação anterior, e tornando-se conhecida também como novo Marco Regulatório do Saneamento Básico. Essa lei acrescenta mais pontos, tais como: a inserção do meio privado em serviços de saneamento, criação de um Comitê Interministerial de Saneamento Básico (CISB), ampliação das atuações da Agência Nacional de Águas (ANA), fim de lixões, responsabilização de serviços e busca da universalização do saneamento até o final de 2033 (BRASIL, 2020).

\section{CONSIDERAÇÕES FINAIS}


A partir das informações e dados levantados, torna-se observável a necessidade da correta implementação de saneamento básico para a população, ressaltando este como elemento fundamental para melhoria da qualidade de vida. Valendo destacar que embora exista uma legislação vigente o seu alvo de atuação dependerá de todos os cidadãos e gestores, o que reitera a necessidade de conscientização social.

\section{REFERÊNCIAS}

1. BRASIL. Lei no 14.026. 2020. Disponível em: http://www.planalto.gov.br/ccivil_03/_ato20192022/2020/lei/l14026.htm. Acessado em 9 de outubro de 2021.

2. BRASIL. SNIS. Sistema Nacional de Informação sobre Saneamento. Painel de informações sobre o Saneamento no Brasil. 2019. Disponível em: http://www.snis.gov.br/painel-informacoes-saneamentobrasil/web/painel-setor-saneamento. Acessado em 9 de outubro de 2021.

3. COSTA RNP, PINHEIRO EM. O Cenário do Saneamento Básico no Brasil. Revista Educação Ambiental em Ação, 2018; 76: 1-26.

4. MELLO MTC, MELLO LTC. As relações dos sistemas de saneamento e a saúde da população: um estudo em um distrito de um município no Rio Grande do Norte. Revista Eletrônica Acervo Saúde, 2019; 11: e1492. 


\title{
O DESENVOLVIMENTO NEUROPSICOMOTOR E A INFLUÊNCIA DE RISCOS BIOLÓGICOS NO CRESCIMENTO INFANTIL
}

\author{
Telma da Silva Amorim ${ }^{1}$ \\ Pedro Jonathan Sousa Araujo ${ }^{2}$ \\ Gislane de Oliveira Souza ${ }^{3}$
}

1. Faculdade leducare (FIED), Tianguá - CE.

2. Universidade Federal do Delta do Parnaíba (UFDPar), Parnaíba - PI.

3. Instituto de Estudos e Pesquisas do Vale do Acarú (IVA). Tianguá - CE.

Palavras-chave: Motricidade, Desenvolvimento, Fatores de risco.

\section{INTRODUÇÃO}

O desenvolvimento humano é um processo contínuo ligado a idade, processo esse em que o indivíduo adquire habilidades psicomotoras transformadas em movimentos. Dessa forma, a exposição durante a primeira infância a fatores biológicos e psicossociais pode afetar diretamente estruturas e funções cerebrais, agindo diretamente sobre o comprometimento do desenvolvimento de crianças, bem como, suas trajetórias de desenvolvimentos futuros (DELGADO DA, et al., 2020).

As experiências da criança na primeira infância são extremamente importantes, pois são capazes de produzir alterações permanentes em seu cérebro. Outrossim, o ambiente tem efeitos rápidos na anatomia cerebral, provocando inúmeras mudanças positivas no nível de aprendizado do infante (CARDOSO KVV, et al., 2021).

\section{OBJETIVO}

Realizar uma revisão integrativa de literatura para analisar e investigar a associação do desenvolvimento neuropsicomotor infantil com as influências do ambiente biológico da criança na primeira infância, assim como, práticas parentais e ambientais que facilitam a motricidade do infante.

\section{MÉTODO}

Trata-se de uma revisão integrativa de literatura, realizada a partir da base de dados SciELO. Foram incluídos artigos em português, publicados entre 2020 e 2021 e disponíveis na íntegra para a leitura, buscados com as palavras-chave: "desenvolvimento motor", "fatores de risco e infância", encontrando um total de 4 artigos para leitura e análise dos dados.

\section{REVISÃO BIBLIOGRÁFICA}


O Desenvolvimento Neuropsicomotor (DNPM) é o processo que os estímulos são essenciais para o desenvolvimento da criança, cujo as habilidades são adquiridas, como os aspectos motores, afetivos, cognitivos e psicomotores. Os fatores biológicos podem influenciar no crescimento da criança positivamente ou negativamente. Assim, condições econômicas são uma das principais causas, pois, a qualidade dos estímulos muitas vezes compromete o desenvolvimento infantil (DEFILIPO ÉC, et al., 2021).

O ambiente pode proporcionar estímulos ou não, um dos agravamentos como o fator biológico, está direcionado a falta de nutrição essencial para a criança desde a gestação até o seu crescimento, causando problemas a outros fatores importantes para o desenvolvimento da criança (AGUIAR CP, et al., 2021).

\section{CONSIDERAÇÕES FINAIS}

Dessa forma, após a análise dos artigos selecionados ficou evidente que, os fatores biológicos influenciam no desenvolvimento neuropsicomotor da criança na aquisição das habilidades em diferentes áreas, assim como uma das principais causas com relação as condições socioeconômicas das famílias.

\section{REFERÊNCIAS}

1. AGUIAR CP, et al. A influência do estímulo e do vínculo familiar no desenvolvimento de crianças com síndrome de Down. Revista Eletrônica Acervo Saúde, 2021; 13(11): 1-7.

2. CARDOSO KVV, et al. Desenvolvimento motor de bebês em intervenção parental durante a puericultura: série de casos. Fisioterapia e Pesquisa, 2021; 28(2): 172-178.

3. DELGADO DA, et al. Avaliação do desenvolvimento motor infantil e sua associação com a vulnerabilidade social. Fisioterapia e Pesquisa,2020; 27(1): 48-56.

4. DEFILIPO ÉC, et al. Oportunidades do ambiente domiciliar e desenvolvimento motor de lactentes no primeiro ano. Fisioterapia em Movimento, 2021; 34: 1-10. 


\title{
CONHECIMENTO DOS UNIVERSITÁRIOS ACERCA DA PANDEMIA DA COVID-19: UMA REVISÃO INTEGRATIVA DA LITERATURA
}

\author{
Michelle Augusta Soares Oliveira ${ }^{1}$ \\ Gabriela Modesto Figueiredo ${ }^{1}$ \\ Leticia Marques de Sousa ${ }^{1}$ \\ Talita de Cassia Raminelli da Silva ${ }^{1}$ \\ Andréia Guedes Oliva Fernandes ${ }^{1}$
}

1. Centro Universitário Euro Americano (UNIEURO) Brasília - DF.

Palavras-chave: COVID-19, Educação superior, Conhecimento.

\section{INTRODUÇÃO}

A doença denominada COVID-19 é causada pelo novo coronavírus (SARS-CoV-2) também denominado como Severe Acute Respiratory Syndrome-Corona Virus-2 (LUCENA JF e SENA JGB, 2020) tem se espalhado rapidamente a nível nacional e mundial sendo hoje considerada uma pandemia (OMS, 2021). Evidências apontam que a avaliação do conhecimento da sociedade relacionado a um assunto específico deve ser analisada em um pequeno espaço de tempo com o objetivo de que os resultados alcançados tragam informação e contribuam para a saúde pública, a exemplo a população, universitária que é de suma importância uma vez que estes estudantes irão atuar como multiplicadores de conhecimento (BAIG M, et al., 2020; SILVA, M, et al., 2021).

\section{OBJETIVO}

Identificar nas publicações nacionais e internacionais, entre os anos de 2020 até 2021, o nível do conhecimento dos estudantes universitários sobre a pandemia do COVID-19 e os fatores relacionados ao nível de conhecimento.

\section{MÉTODO}

Revisão integrativa, na qual o levantamento das publicações aconteceu nas bases de dados: PUBMED, SCIELO e BVS. Foram incluídos artigos em português, inglês e espanhol, originais, publicados entre 2020 e 2021 e disponíveis na íntegra. A pesquisa resultou em 2.288 artigos dos quais após aplicação dos critérios de elegibilidade resultou em 07 artigos. Foram utilizados os descritores: "Pandemia"; "COVID-19"; "Estudantes"; "Educação Superior" e "Conhecimento" e os operadores booleanos "AND" e "OR".

\section{REVISÃO BIBLIOGRÁFICA}


Foi evidenciado nos estudos selecionados, um nível satisfatório de conhecimento entre os estudantes universitários acerca da pandemia da COVID-19, dentre os fatores que contribuem para o conhecimento satisfatório sobre os diversos aspectos da pandemia na população universitária estão o gênero feminino e o nível de escolaridade. Reconhece-se que o conhecimento satisfatório sobre a COVID-19 possibilita a maior adesão às medidas de prevenção e controle da mesma, além de promover comportamentos positivos com incentivo de educar outras pessoas e expandir o conhecimento sobre a situação atual em todo o mundo (BAIG M, et al.; 2020; NOREEN K, et al., 2020).

\section{CONSIDERAÇÕES FINAIS}

A avaliação do conhecimento entre estudantes universitários é crucial, pois oportuniza a identificação das facilidades e dificuldades que os mesmos têm a respeito desta doença infecciosa. A partir disto, é possível pensar em estratégias de ensino aprendizagem que possibilitem sanar as dificuldades/desafios encontrados acerca do conhecimento e assim reduzir a transmissão da enfermidade além do fato destes terem a possibilidade de serem multiplicadores do conhecimento em distintos ambientes.

\section{REFERÊNCIAS}

1. BAIG M, et al. Preditores de equívocos, conhecimentos, atitudes e práticas da pandemia de COVID-19 entre uma amostra da população saudita. PLoS One, 2020; 15(12).

2. LUCENA JF, SENA JGB. Residência Integrada Multiprofissional em Saúde e a pandemia COVID-19: um relato de experiência. Revista Eletrônica Acervo Saúde, 2020; 12(9): e4964.

3. NOREEN K, et al. Conhecimento, atitudes e práticas contra a crescente ameaça do COVID-19 entre estudantes de medicina do Paquistão. PLoS One, 2020; 15(12).

4. ORGANIZAÇÃO MUNDIAL DA SAÚDE (OMS). Folha informativa COVID-19. Escritório da OPAS e da OMS no Brasil. Brasil, 2021. Disponível em: https://www.paho.org/pt/covid19. Acessado em: 24 de setembro de 2021.

5. SILVA M, et al. Coronavírus: consequências da pandemia no ensino superior. Revista de Enfermagem e Atenção à Saúde, 2021; 13(5). 


\title{
POTENCIAL TECNOLÓGICO DAS NANOPARTÍCULAS MAGNÉTICAS COMO PRÁTICA ECOLÓGICA NO TRATAMENTO DE ÁGUA
}

\author{
Elizeu Sousa Lira ${ }^{1}$
}

Rafael Leal da Silva ${ }^{1}$

1. Escola Cidadã Integral Técnica Alfredo Pessoa de Lima, Solânea - PB.

Palavras-chave: Nanopartículas, Agroecologia, Ensino médio.

\section{INTRODUÇÃO}

No século XXI, existe a necessidade de inovações que colaborem para o desenvolvimento sustentável dos pilares socioeconômicos. Nesse sentido, as práticas agroecológicas definem um degrau que possa servir de apoio para notoriedade de tal via, ou seja, com acoplamento de técnicas agroecológicas de produção, objetivada ao tratamento de água juntamente com a utilização de nanopartículas magnéticas, visto que a influência da nanociência e nanotecnologia estão em destaque nas áreas afins, atualmente (ROSA VC e CAMPOS GS, 2020). Nesse sentido, abordaremos a perspectiva da Ciência, Tecnologia, Sociedade e Ambiente (CTSA), como fator transversal e interdisciplinar (TONET MD e LEONEL AA, 2019).

\section{OBJETIVO}

Apresentar um meio para o tratamento de água a partir de uma revisão de literatura científica sobre nanotecnologia e princípios agroecológicos sustentáveis, visando estabelecer a importância da nanotecnologia na sociedade contemporânea.

\section{REVISÃO BIBLIOGRÁFICA}

O fator inovação tem sido o foco das pesquisas em sustentabilidade, por isso é importante se sustentar em novos processos que seja replicável em escala de Ensino Básico (TONET MD e LEONEL AA, 2019). Nessa esfera, o controle das nanopartículas magnéticas nos proporciona um potencial de agregar conhecimento para o tratamento de água a longo prazo, o que culmina na inovação social sustentável e ambiental (SANTOS TRT, et al., 2019). Entre as técnicas mais viáveis para produção de nanopartículas que pode ser replicado em laboratórios de Ensino Básico se destaca o método de reação por coordenação iônica usando o biopolímero quitosana (SILVA RL, 2020).

Além disso, o acoplamento das técnicas agroecológicas fornece um mecanismo de efetivação do projeto, no qual o olhar é direcionado para o próprio desenvolvimento sustentável (ROSA VC e CAMPOS GS, 2020). As nanopartículas magnéticas, além de proporcionarem uma solução plausível para sociedade, dentro do contexto CTSA, assim, sendo de baixo custo e totalmente viável (SANTOS TRT, et al., 2019). Diante do exposto, o acoplamento da agroecologia com a nanotecnologia para o tratamento de água é uma proposta viável. 


\section{CONSIDERAÇÕES FINAIS}

Com a realização da presente pesquisa bibliográfica, foi possível observar a pertinência nos desenvolvimentos e caracterizações das nanopartículas magnéticas para o tratamento de água, isso diante das técnicas acopladas referente a agroecologia e nanotecnologia, no qual confirmou o real vínculo de técnicas aplicadas na escala nanométrica visada. Essas técnicas, que são focadas na despoluição da água para o reuso, são aprovadas, afirmando assim, tal relevância social, ambiental e científica.

\section{REFERÊNCIAS}

1. ROSA VC, CAMPOS GS. A agroecologia como mecanismo de efetivação dos objetivos de desenvolvimento sustentável no brasil. Revista Eletrônica da Faculdade de Direito de Franca, 2020; 15(1), 321-340.

2. SANTOS TRT, et al. Aplicação da nanotecnologia no tratamento de água: uma revisão. Revista Uningá review, 2019; 34(2), 51-72.

3. SILVA RL. Caracterização estrutural de nanopartículas de ferrita de cobalto dopadas com La3+ para aplicação em tratamento de água. Revista Eletrônica Acervo Saúde, 2020; 54: e4649.

4. TONET MD, LEONEL AA. Nanociência e Nanotecnologia: uma revisão bibliográfica acerca das contribuições e desafios para o ensino de Física. Caderno Brasileiro de Ensino de Física, 2019; 36(2), 431 456. 


\title{
ENTENDENDO O FENÔMENO RELIGIOSO SEGUNDO VIKTOR FRANKL
}

\author{
Tiago Mota Ferreira ${ }^{1}$ \\ Alberlene Baracho Sales ${ }^{1}$ \\ Maura Rahianny Cardoso Araújo ${ }^{1}$ \\ Marciele de Lima Silva ${ }^{1}$ \\ Thiago Antonio Avellar de Aquino ${ }^{1}$
}

1. Universidade Federal da Paraíba (UFPB), João Pessoa - PB.

Palavras-chave: Viktor Frankl, Fenomenologia, Espiritualidade.

\section{INTRODUÇÃO}

Viktor Frankl fala a respeito do ser humano como um ser tridimensional compreendido em seus fenômenos físicos, psicológicos e dimensão noética (nous-espírito). O autor aborda algumas divergências entre suas perspectivas em relação a espiritualidade e religiosidade, e as dos psicanalistas de destaque, Freud e Jung. Viktor Frankl, neurologista e psiquiatra, descreve em suas teorias a importância da psicologia da religião. É o criador da Logoterapia, que consiste em três fundamentos principais: a liberdade da vontade (liberdade de tomar qualquer atitude), a vontade de sentido (o indivíduo não deve ser guiado pelo princípio do prazer ou poder) e o sentido da vida (conceito de liberdade e responsabilidade) (AQUINO TAA, et al., 2019).

\section{OBJETIVO}

Analisar a perspectiva de Frankl referente ao fenômeno religioso, considerando os aspectos metodológicos da fenomenologia das religiões. Considerando as prerrogativas de como seus escritos podem contribuir para esse olhar fenomenológico.

\section{REVISÃO BIBLIOGRÁFICA}

Nesta pesquisa bibliográfica de âmbito narrativo-descritivo, buscou-se na literatura de referência as percepções fenomenológicas de Viktor Frankl, e o estudo sobre o ser humano que o mesmo descreve assertivamente. Foram analisados livros e publicações em revistas de referência que associam o Viktor Frankl a temática da fenomenologia, sob a ênfase do fenômeno religioso. A fenomenologia é uma ciência em declínio que serve muito aos interesses teológicos, mas para as estruturas do saber científico deixa a desejar e, portanto, seu declínio tem em sua própria insuficiência a razão para não fazer parte significativa dos estudos científicos (JUNIOR VA, 2017).

Para Frankl, o ser humano além de ter seu inconsciente referente ao seu psicológico, também é dotado de uma espiritualidade inconsciente. $\mathrm{O}$ autor apresenta a religiosidade latente em si, o que ele chamou de presença ignorada de Deus. Essa dimensão noológica, ou espiritualidade inconsciente, ou até mesmo este 
inconsciente transcendental, não se refere apenas ao aspecto da religiosidade, mas também da intelectual e artístico (AQUINO TAA, et al., 2020).

\section{CONSIDERAÇÕES FINAIS}

A consciência como fator psicológico é imanente do ser humano, ela nos remete por si mesma a transcendência que buscamos na religião. $O$ ser humano religioso também tem consciência e responsabilidade, mas por ignorar a transcendência da consciência não sei conversa com ela, não se pergunta pelo que é responsável ou de onde ela vem.

\section{REFERÊNCIAS}

1. AQUINO TAA, et al. Monantropismo e Movimento para a paz no Pensamento de Viktor Frankl. Revista Interações, 2019; 14(26).

2. CRUZ JS, AQUINO TAA. Espiritualidade e resiliência: relevância e implicações no pensamento frankliano. REVER-Revista de Estudos da Religião, 2020; 20(2): 89-103.

3. JÚNIOR VA. "Alegria e Tremor ao Mesmo Tempo": Alguns Aspectos Simbólicos do Numinoso em um Hino Clássico do Protestantismo. Último Andar, 2017; 30: 185-200. 


\title{
| RELATOS DE EXPERIÊNCIA
}

RESUMO SIMPLES: Relato de Experiência

\section{IMPLANTAÇÃO DO CURSO DE PEDAGOGIA MODALIDADE A DISTÂNCIA PELA UNIVERSIDADE FEDERAL DO TRIÂNGULO MINEIRO: UM RELATO DE EXPERIÊNCIA}

\author{
Franciely Alves da Silva ${ }^{1}$ \\ Leandro Garcia Resende ${ }^{1}$ \\ Mateus Beordo ${ }^{1}$ \\ Laércio de Jesus ${ }^{1}$
}

1. Universidade Federal do Triângulo Mineiro (UFTM), Uberaba - MG.

Palavras-chave: Educação a distância, Ensino superior, Pedagogia.

\section{INTRODUÇÃO}

A sociedade está em constante transformação, e a educação precisa acompanhar essas transformações (SILVA DV, 2021). Com a polarização da internet entre 1997 e 2005 no Brasil, ocorreu uma expansão da educação a distância (EAD), permitindo novas possibilidades e vivências para discentes e docentes (SANTOS MR, 2020).

A EAD é uma modalidade de ensino pode possibilitar formas diferentes de ver o mundo, de ensinar e aprender, dessa forma é necessário que mais políticas públicas educacionais sejam implementadas buscando estabelecer legislações específicas de incentivo a programas de EAD (RIBEIRO MA e SILVA SJ, 2020).

\section{OBJETIVO}

Relatar a experiência dos discentes em relação a instalação do curso de pedagogia a distância pela Universidade Federal do Triângulo Mineiro, Uberaba- MG. Os pontos levantados se deram pelas experiências dos autores durante os primeiros períodos do curso.

\section{RELATO DE EXPERIÊNCIA}

O curso de pedagogia da Universidade Federal do Triângulo Mineiro (UFTM), iniciou-se com 200 alunos distribuídos em 5 polos, atualmente composto por 181 alunos. Observamos, que não houve uma queda drástica do curso ofertado gratuitamente. Em diálogos com os alunos entre si por meio de reuniões de grupos ou por meio de grupos de WhatsApp, percebemos que, de início, umas das principais dificuldades diz respeito a ambientação do tipo de ensino proposto como também da plataforma de ensino (Moodle). 
Outro tipo de dificuldade é a necessidade da relação professor-aluno que, por ventura, está cada vez mais sendo sanada através da própria plataforma do Moodle ofertado pela universidade. Outro ponto importante que deve ser destacado é a importância desse tipo de ensino EAD, uma vez que, maior parte dos alunos não possuem condições financeiras para custear um ensino superior; além de que, o EAD possui um período mais flexível de horário, permitindo que maior parte dos discentes possam estudar e trabalhar.

\section{CONSIDERAÇÕES FINAIS}

Diante dos relatos obtidos foi possível perceber que embora os alunos obtiveram e ainda possuem dificuldades no curso de pedagogia ofertado na modalidade EAD pela UFTM, é inegável a sua importância como metodologia de ensino que permite a formação de cidadãos que perante a sociedade fara sua diferença, além de contribuir significativamente para a democratização do acesso ao ensino superior nas mais distantes cidades do país.

\section{REFERÊNCIAS}

1. SILVA DV. Educação e novas tecnologias: um (re) pensar. Caderno Intersaberes, 2021; 10(26): 181-194.

2. SANTOS SMR, et al. Docência superior na formação EAD no curso de enfermagem: Revisão bibliográfica. Revista Eletrônica Acervo Científico, 2020; 8: e2204.

3. RIBEIRO MA, SILVA SJ. Programas de Fomento nos cursos de Licenciatura na Modalidade EaD: políticas públicas para formação docente. Revista Educação e Políticas em Debate, 2020; 9(1): 169-186. 
RESUMO SIMPLES: Relato de Experiência

\title{
A APLICABILIDADE RENTÁVEL DAS ESTACAS PRÉ-MOLDADAS DE CONCRETO ARMADO
}

\author{
Allefy Teles Sampaio ${ }^{1}$ \\ Jéssica Wanderley Souza do Nascimento² \\ Sabiana Gilsane Mühlen dos Santos²
}

1. Universidade de Fortaleza (UNIFOR), Fortaleza - CE.

2. Universidade Federal de Santa Maria (UFSM), Santa Maria - RS.

Palavras-chave: Estaca raiz, Estaca cravada, Fundação.

\section{INTRODUÇÃO}

A natureza heterogênea dos solos de fundação e a sua grande variabilidade natural é algo que move grandes discussões, na prática da engenharia, não sendo ainda possível o seu pleno entendimento (LUCIANI $A$ e $D E L C D, 2018$ ). Ao longo dos anos foram propostos vários métodos para obter informações tanto sobre a sua composição quanto sobre o seu comportamento, tais como a utilização frequente de metodologias predominantemente empíricas e semi-empíricas (SONDA D, et al., 2019).

Contudo, ainda é comum a necessidade de modificar a concepção das estruturas de fundação quando as condições do solo diferem das esperadas, especialmente quando não foram realizadas investigações aprofundadas no subsolo (ARAUJO WS, et al., 2018).

\section{OBJETIVO}

Externar as causas pelas quais foi realizada a alteração do método construtivo de estaca raiz para estaca pré-moldada de concreto armado do projeto inicial da sede do novo Centro Educacional de Tempo Integral (CETI) na cidade de Tabatinga/AM.

\section{RELATO DE EXPERIÊNCIA}

O presente relato enfoca um caso real como alternativa para a utilização de um método construtivo das fundações que contorna as exigências do projeto inicial. Dessa forma, após várias análises e estudos realizados sobre o assunto verificou-se o elevado risco técnico de falha durante seu processo executivo devido à baixa resistência do solo. Assim, tornando necessária adotar outro modelo de metodologia, onde se optou pelo método de fundações de estacas de concreto armado pré-moldadas.

Com a adoção dessa mudança, pesquisadores afirmam que será obtido um melhor controle tecnológico durante a realização tanto das estacas como da cravação, acarretando para a obra apenas vantagens como: rapidez de execução, alta segurança, custo reduzido, aumento na produtividade mesmo em período chuvoso, baixo consumo de água e um canteiro mais limpo, quando comparada a fundação anterior. 
Convém lembrar que a análise apresentada, com a sugestão de mudança, visa apenas garantir a integridade do trabalho, de modo a otimizar os prazos contratados, bem como a adaptação às características do terreno, da melhor maneira possível.

\section{CONSIDERAÇÕES FINAIS}

Em virtude dos fatos mencionados, mostram-se que a solução originalmente proposta não é possível de ser executada, consequentemente correndo o risco de não gerar o resultado esperado, onde só poderá ser avaliado após a execução, ocasionando possíveis infortúnios ao planejamento físico-financeiro da obra.

\section{REFERÊNCIAS}

1. ARAUJO WS, et al. Análise e dimensionamento de fundações profundas, visando a viabilidade econômica e segurança estrutural. Revista Científica Multidisciplinar Núcleo do Conhecimento, 2018; 12(8): $119-143$.

2. LUCIANI A, DEL CD. Towards a resilient perspective in building conservation. Journal of Cultural Heritage Management and Sustainable Development, 2018; 8(3): 309-320.

3. SONDA D, et al. The restoration and seismic strengthening of the earthquake-damaged UNESCO heritage palace in Kathmandu. International Journal of Architectural Heritage, 2019; 13(1): 153-171. 


\section{AGRADECIMENTOS}

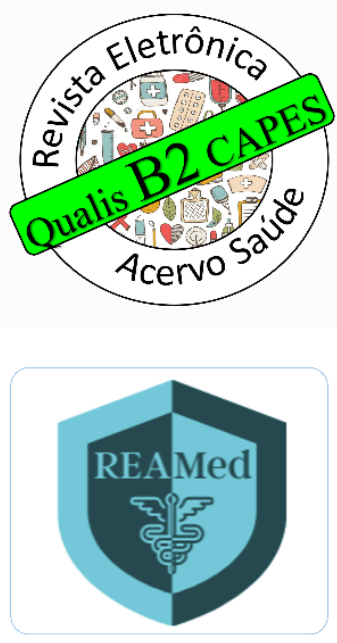

\section{Revista}

Eletrônica

Acervo

Saúde

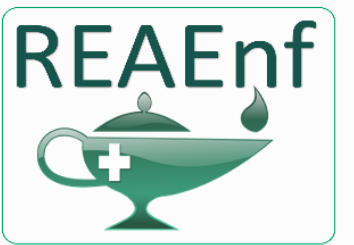

Revista

Eletrônica

Acervo

Enfermagem

REAQdonto

Revista

Eletrônica

Acervo

Odontológico

\section{$R E A C$ Revista \\ Eletrônica \\ Acervo \\ CIENTífico}

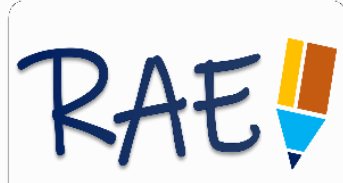

Revista

Acervo

Educacional

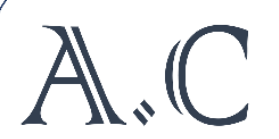

Revista

Artigos.

Com 